
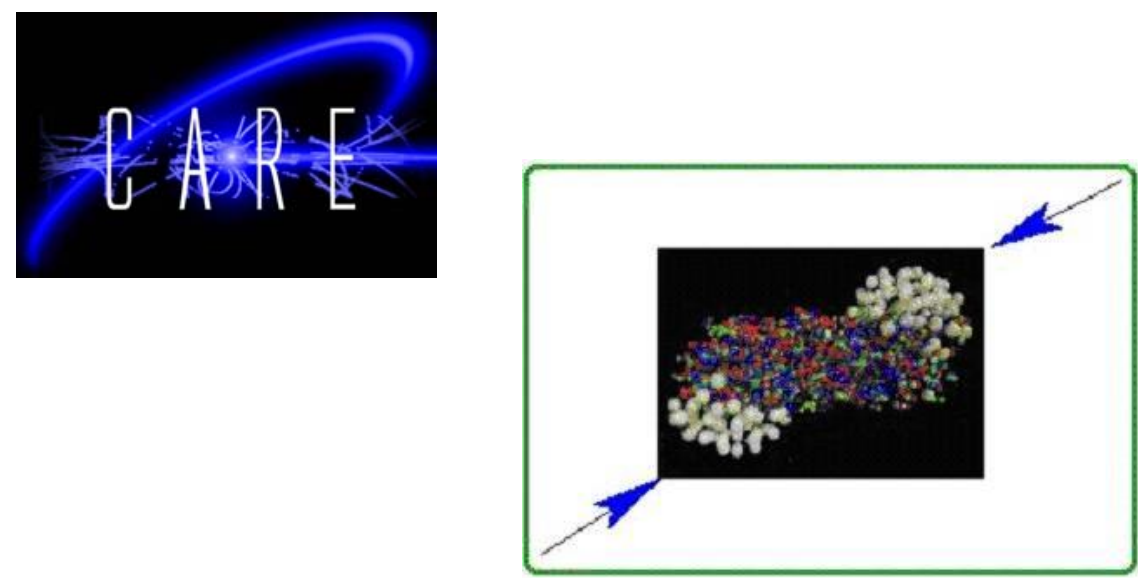

High Energy

High Intensity

Hadron Beams

\title{
Scenarios for sLHC and vLHC
}

\author{
W. Scandale ${ }^{1}$, F. Zimmermann ${ }^{1}$
}

1) CERN, Geneva, Switzerland

\begin{abstract}
The projected lifetime of the LHC low-beta quadrupoles and evolution of the statistical error halving time call for an LHC luminosity upgrade by the middle of the coming decade. In the framework of the EU CAREHHH network, two scenarios have been developed for increasing the LHC peak luminosity by a factor 10 , to $10^{35} \mathrm{~cm}^{-2} \mathrm{~s}^{-1}$ ("sLHC"). Both scenarios imply a rebuilding of the high-luminosity interaction regions (IRs) in combination with a consistent change of beam parameters. However, their respective features, bunch structures, IR layouts, merits and challenges differ substantially. In either scenario luminosity leveling during a store would be advantageous for the physics experiments. Longer-term R\&D efforts are devoted to a higher-energy hadron collider ("vLHC"), which could be realized on a green field or as a later and more radical LHC upgrade.
\end{abstract}

Contribution to the "Hadron Collider Physics Symposium HCP 2007”, Elba (Italy), 20-26 May 2007

Work supported by the European Community-Research Infrastructure Activity under the FP6 "Structuring the European Research Area" programme (CARE, contract number RII3-CT2003-506395) 


\title{
Scenarios for sLHC and vLHC
}

\author{
W. Scandale ${ }^{\mathrm{a} *}$, F. Zimmermann ${ }^{\mathrm{a}}$. \\ ${ }^{\mathrm{a}} \mathrm{CERN}, 1211$ Geneva 23, Switzerland
}

The projected lifetime of the LHC low-beta quadrupoles and evolution of the statistical error halving time call for an LHC luminosity upgrade by the middle of the coming decade. In the framework of the EU CAREHHH network, two scenarios have been developed for increasing the LHC peak luminosity by a factor 10, to $10^{35} \mathrm{~cm}^{-2} \mathrm{~s}^{-1}$ ("sLHC"). Both scenarios imply a rebuilding of the high-luminosity interaction regions (IRs) in combination with a consistent change of beam parameters. However, their respective features, bunch structures, IR layouts, merits and challenges differ substantially. In either scenario luminosity leveling during a store would be advantageous for the physics experiments. Longer-term R\&D efforts are devoted to a higher-energy hadron collider ("vLHC"), which could be realized on a green field or as a later and more radical LHC upgrade.

\section{INTRODUCTION}

The Large Hadron Collider (LHC) will collide two proton beams with a centre-of-mass energy of $14 \mathrm{TeV}$ at design and ultimate luminosities of $10^{34} \mathrm{~cm}^{-2} \mathrm{~s}^{-1}$ and $2.3 \times 10^{34} \mathrm{~cm}^{-2} \mathrm{~s}^{-1}$. The LHC proton beams will cross each other at the four detectors of the two high-luminosity experiments ATLAS and CMS, the B physics experiment LHC-B, and the ion experiment ALICE.

Simple models for the LHC luminosity evolution over the first few years of operation [1] indicate that the IR quadrupoles may not survive for more than 8 years due to high radiation doses, and that already after $4-5$ years of operation the halving time of the statistical error will exceed 5 years. Either consideration stresses the need for an LHC luminosity upgrade around 2015. This upgrade could consist of two stages, the first one consolidating the nominal performance and providing a luminosity of up to $3 \times 10^{34} \mathrm{~cm}^{-2} \mathrm{~s}^{-1}$ and the second one increasing the luminosity to ten times the nominal, i.e. to $10^{35} \mathrm{~cm}^{-2} \mathrm{~s}^{-1}$ Possible LHC upgrade paths, first examined around 2001 [2], are being further developed by the CARE [3] $\mathrm{HHH}$ network [4], in collaboration with the US LARP [5].

*We acknowledge the support of the European Community-Research Infrastructure Initiative under the FP6 "Structuring the European Research Area" programme (CARE, contract number RII3-CT-2003-506395)

\section{BEAM PARAMETERS}

Near the beam-beam limit with alternating planes of crossing at two interaction points (IPs), the luminosity can be expressed as $[6]$

$L \approx \frac{f_{\text {rev } \gamma}}{2 r_{p}} n_{b} \frac{1}{\beta^{*}} N_{b} \Delta Q_{b b} F_{\text {profile }} F_{\mathrm{hg}}$,

where $\Delta Q_{b b}$ denotes the total beam-beam tune shift, limited to about 0.01 according to experience at previous hadron colliders, $f_{\text {rev }}$ the revo-

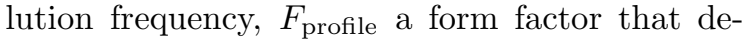
pends on the longitudinal profile (equal to 1 for a Gaussian and $\sqrt{2}$ for a uniform profile) and $F_{\mathrm{hg}}$ the reduction factor due to the hourglass effect, which is relevant for bunch lengths comparable to, or smaller than, the IP beta function. Collision of round beams is assumed. Other variables are defined in Table 1, which compares parameters for the nominal and ultimate LHC with those for two upgrade scenarios (abbreviated "ES" and "LPA"). The upgrade parameters in (1) which differ from the ultimate LHC configuration are $\beta^{*}, N_{b}, \Delta Q_{b b}, F_{\text {profile }}$ and $n_{b}$ for LPA, and $\beta^{*}$, $\Delta Q_{b b}$ and $F_{\mathrm{hg}}$ in the ES scheme.

In the "early-separation" (ES) scenario [7] one stays with the ultimate LHC beam, squeezes $\beta^{*}$ down to about $10 \mathrm{~cm}$ in ATLAS and CMS; and adds early-separation dipoles inside the detectors starting at about $3 \mathrm{~m}$ from the IP. Optionally, ES could also include a quadrupole doublet at 
Table 1

Parameters for the (1) nominal and (2) ultimate LHC compared with those for the two upgrade scenarios with (3) more strongly focused ultimate bunches with early separation at 25-ns spacing [ES], (4) longer intense flat bunches at 50-ns spacing in a regime of large Piwinski angle [LPA]. The numbers refer to the performance without luminosity leveling.

\begin{tabular}{|lccccc|}
\hline parameter & symbol & nominal & ultimate & ES & LPA \\
\hline number of bunches & $n_{b}$ & 2808 & 2808 & 2808 & 1404 \\
protons per bunch & $N_{b}\left[10^{11}\right]$ & 1.15 & 1.7 & 1.7 & 4.9 \\
bunch spacing & $\Delta t_{\text {sep }}[\mathrm{ns}]$ & 25 & 25 & 25 & 50 \\
average current & $I[\mathrm{~A}]$ & 0.58 & 0.86 & 0.86 & 1.22 \\
normalized transverse emittance & $\gamma \epsilon[\mu \mathrm{m}]$ & 3.75 & 3.75 & 3.75 & 3.75 \\
longitudinal profile & & Gaussian & Gaussian & Gaussian & uniform \\
rms bunch length & $\sigma_{z}[\mathrm{~cm}]$ & 7.55 & 7.55 & 7.55 & 11.8 \\
beta function at IP1\&5 & $\beta^{*}[\mathrm{~m}]$ & 0.55 & 0.5 & 0.08 & 0.25 \\
(effective) crossing angle & $\theta_{\mathrm{c}}[\mu \mathrm{rad}]$ & 285 & 315 & 0 & 381 \\
Piwinski angle & $\phi$ & 0.4 & 0.75 & 0 & 2.01 \\
hourglass factor & $F_{\mathrm{hg}}$ & 1.00 & 1.00 & 0.86 & 0.99 \\
peak luminosity & {$\left[10^{34} \mathrm{~cm}-2 \mathrm{~s}^{-1}\right]$} & 1.0 & 2.3 & 15.5 & 10.6 \\
events per crossing & & 19 & 44 & 294 & 403 \\
rms length of luminous region & $\sigma_{\mathrm{lum}}[\mathrm{mm}]$ & 45 & 43 & 53 & 37 \\
initial luminosity lifetime & $\tau_{\mathrm{L}}[\mathrm{h}]$ & 22.2 & 14.3 & 2.2 & 4.5 \\
average luminosity $\left(T_{\mathrm{ta}}=10 \mathrm{~h}\right)$ & $L_{\mathrm{av}}\left[10^{34} \mathrm{~cm}{ }^{-2} \mathrm{~s}^{-1}\right]$ & 0.5 & 0.9 & 2.4 & 2.5 \\
optimum run time $\left(T_{\mathrm{ta}}=10 \mathrm{~h}\right)$ & $T_{\mathrm{run}}[\mathrm{h}]$ & 21.2 & 17.0 & 6.6 & 9.5 \\
average luminosity $\left(T_{\mathrm{ta}}=5 \mathrm{~h}\right)$ & $L_{\mathrm{av}}\left[10^{34} \mathrm{~cm}{ }^{-2} \mathrm{~s}^{-1}\right]$ & 0.6 & 1.2 & 3.6 & 3.5 \\
optimum run time $\left(T_{\mathrm{ta}}=5 \mathrm{~h}\right)$ & $T_{\mathrm{run}}[\mathrm{h}]$ & 15.0 & 12.0 & 4.6 & 6.7 \\
e-cloud heat load for $\delta_{\text {max }}=1.4$ & $P_{\mathrm{ec}}[\mathrm{W} / \mathrm{m}]$ & 1.07 & 1.04 & 1.0 & 0.4 \\
e-cloud heat load for $\delta_{\text {max }}=1.3$ & $P_{\mathrm{ec}}[\mathrm{W} / \mathrm{m}]$ & 0.44 & 0.6 & 0.6 & 0.1 \\
SR heat load & $P_{\mathrm{SR}}[\mathrm{W} / \mathrm{m}]$ & 0.17 & 0.25 & 0.25 & 0.36 \\
image-current heat load & $P_{\mathrm{ic}}[\mathrm{W} / \mathrm{m}]$ & 0.15 & 0.33 & 0.33 & 0.70 \\
\hline
\end{tabular}

about $13 \mathrm{~m}$ from the IP [8]. The ES scenario implies installation of new hardware inside the ATLAS and CMS detectors, as well as, most likely, the first ever hadron beam crab cavities. The latter would gain a factor 2 to 5 in luminosity [7] by ensuring an effective Piwinski angle equal to zero. Their presence is assumed in Table 1. The maximum bunch intensity $N_{b}$ is linked to the limit on the total beam-beam tune shift for two IPs, via $\left|\Delta Q_{b b}\right|=N_{b} r_{p} \beta^{*} /\left(2 \pi \gamma \sigma^{* 2}\right)$, where $\sigma^{*}$ denotes the transverse rms beam size at the IP. A corresponding IR layout is sketched in Fig. 1. Its merits are the negligible effect of most longrange collisions thanks to the early separation, the absence of any geometric luminosity loss except for the hourglass effect, and no increase in the beam current beyond ultimate. Challenges include the early separation dipoles 'D0' deep inside the detector, the optional s.c. quadrupole doublet 'Q0', also embedded, strong larger-aperture low- $\beta$ quadrupoles based on $\mathrm{Nb}_{3} \mathrm{Sn}$, use of crab cavity for hadron beams [9], the remaining 4 parasitic collisions at $4-5 \sigma$ separation, a significant off-momentum beta beating which may degrade collimation efficiency, plus low beam and luminosity lifetimes (proportional to $\beta^{*}$ ).

In the "large Piwinski angle" (LPA) scenario the bunch spacing is doubled, to $50 \mathrm{~ns}$; longer, longitudinally flat, and more intense bunches are collided with a large Piwinski angle of $\phi \equiv \theta_{c} \sigma_{z} /\left(2 \sigma^{*}\right) \approx 2$; the IP beta function is reduced by a more moderate factor of 2 
to $\beta^{*} \approx 25 \mathrm{~cm}$; and long-range beam-beam wire compensators [10] are installed upstream of the inner triplets. This regime of large $\phi$ and uniform bunch profile allows raising the bunch intensity $N_{b}$ in (1) and thereby the luminosity, since lengthening the bunches in proportion to $N_{b}$ maintains a constant value of $\Delta Q_{b b}$. Figure 2 illustrates the IR layout for this upgrade option. Its merits are the absence of accelerator elements inside the detector, no crab cavities, reduced IR chromaticity, and relaxed IR quadrupoles (for $\beta^{*} \approx 25 \mathrm{~cm}$ various possible optics solutions based on largeaperture NbTi quadrupoles exist [11]; though the survival of the latter at high luminosity still remains to be demonstrated). Challenges are the operation with large Piwinski angle, unproven for hadron beams, the high bunch charge, in particular the beam production and acceleration through the SPS, the larger beam current, and, lastly, the (almost established) wire compensation.

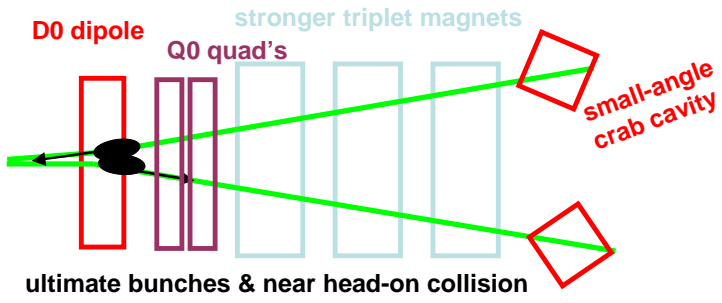

Figure 1. Possible interaction-region layout for the early-separation (ES) scheme, with highly squeezed optics $\left(\beta^{*} \approx 8 \mathrm{~cm}\right)$.

Figure 3 compares the luminosity evolution for the two scenarios. A turn-around time (the time between the end of a collision run and the start of the next collisions) of $5 \mathrm{~h}$ and the corresponding optimum run durations from Table 1 are assumed. The dashed lines indicate the respective time-averaged luminosities. The instantaneous luminosity decays as $L(t)=\hat{L} /\left(1+t / \tau_{\text {eff }}\right)^{2}$ with $\tau_{\text {eff }} \equiv n_{b} N_{b}(0) /\left(\hat{L} \sigma_{\text {tot }} n_{I P}\right)$ the effective beam lifetime due to burn-off at the collision points,

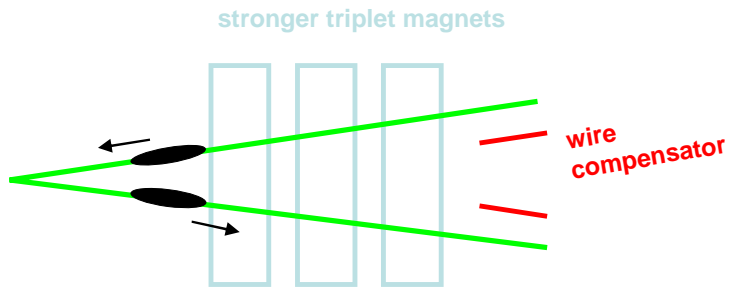

long bunches \& nonzero crossing angle \& wire compensation

Figure 2. Interaction-region layout for largePiwinski-angle (LPA) upgrade with an IP beta function of $0.25 \mathrm{~m}$.

$\sigma_{\text {tot }} \approx 100 \mathrm{mb}$ the relevant total cross section, $n_{I P}$ the number of IPs, and $\hat{L}$ the initial peak luminosity. The optimum average luminosity is $\left.L_{\mathrm{ac}}=\hat{L} \tau_{\mathrm{eff}} /\left(\tau_{\mathrm{eff}}^{1 / 2}+T_{\mathrm{ta}}^{1 / 2}\right)^{2}\right)$, where $T_{t a}$ denotes the turn-around time. The optimum run time $T_{\text {run }}$ is the geometric mean of effective lifetime and turn-around time: $T_{\text {run }}=\sqrt{\tau_{\text {eff }} T_{\text {ta }}}$. In Fig. 3 it can be seen that the luminosity for the ES scenario starts higher, but decays faster than for the LPA case, leading to shorter runs. The average luminosity values are nearly identical. The high initial peak luminosity for ES may not be useful for physics in view of possibly required set-up and tuning periods. On the other hand, the average event pile up for the ES option is about $30-40 \%$ lower than that for the LPA case.

Either upgrade scenario may be adapted for crab-waist collisions [12] by operating with flat beams with $\beta_{x} \gg \beta_{y}$, which would also make optimum use of the available aperture in the lowbeta quadrupoles [13].

The $\mathrm{LHCb}$ detector is special due to its asymmetric location. In the case of LPA, LHCb runs are still possible by adding low-charge satellite bunches in between the main bunches. Otherwise, LHCb would have no collisions. For the ES scenario, colliding beams at LHCb during the second half of each store at enlarged beta function is an option which may also be compatible with the two high-luminosity experiments. 


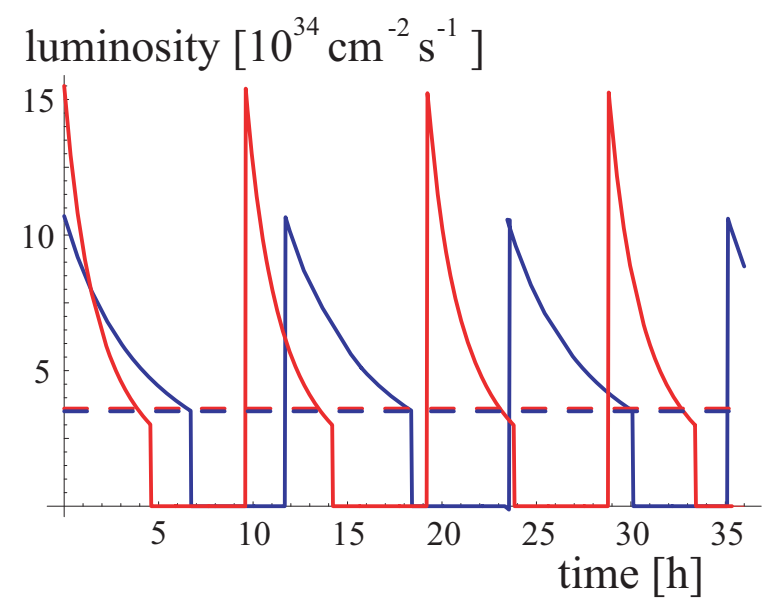

Figure 3. Ideal luminosity evolution for the ES (red) and LPA scenarios (blue), considering the optimum run duration for a turn-around time of $5 \mathrm{~h}$. The dashed lines indicate the corresponding time-averaged luminosities.

\section{LUMINOSITY LEVELING}

Smaller pile up at the start of a physics run, and higher luminosity at the end of each run would be desirable. Such luminosity leveling could be accomplished by dynamic $\beta^{*}$ squeeze, crossing angle variation [14] or changes in the crab rf voltage for ES, and equally by dynamic $\beta$ * squeeze or via bunch-length reduction for LPA.

Leveling provides a constant luminosity, equal to $L_{0}$, and the beam intensity then decreases linearly with time $t$ as $N_{b}=N_{b 0}-L_{0} \sigma_{\text {tot }} n_{I P} / n_{b} t$. The accessible intensity range $\Delta N_{b, \max }$ is limited, e.g., by the range of the leveling variable, for example by the minimum value of $\beta^{*}$, so that the length of a run amounts to $T_{\text {run }}=\Delta N_{b, \max } n_{b} /\left(L_{0} \sigma_{\text {tot }} n_{I P}\right)$, and the average luminosity with leveling becomes $L_{\mathrm{av}, \text { lev }}=$ $L_{0} /\left(1+\Delta N_{b, \max } n_{b} T_{t a} /\left(L_{0} \sigma_{\text {tot }} n_{I P}\right)\right)$. Table 2 compares event rates, run times, and average luminosity values achievable in ES and LPA. In case of $\beta^{*}$ variation, the tune shift decreases during the store, while when leveling via the bunch length or
Table 2

Event rate, run time, and average luminosity for the two upgrade scenarios with leveling.

\begin{tabular}{|lcc|}
\hline & ES & LPA \\
\hline events/crossing & 300 & 300 \\
optimum run time & $\mathrm{N} / \mathrm{A}$ & $2.5 \mathrm{~h}$ \\
av. luminosity $\left[10^{34} \mathrm{~cm}^{-2} \mathrm{~s}^{-1}\right]$ & $\mathrm{N} / \mathrm{A}$ & 2.6 \\
\hline events/crossing & 150 & 150 \\
optimum run time & $2.5 \mathrm{~h}$ & $14.8 \mathrm{~h}$ \\
av. luminosity $\left[10^{34} \mathrm{~cm}^{-2} \mathrm{~s}^{-1}\right]$ & 2.6 & 2.9 \\
\hline events/crossing & 75 & 75 \\
optimum run time & $9.9 \mathrm{~h}$ & $26.4 \mathrm{~h}$ \\
av. luminosity $\left[10^{34} \mathrm{~cm}^{-2} \mathrm{~s}^{-1}\right]$ & 2.6 & 1.7 \\
\hline
\end{tabular}

crossing angle the tune shift increases. For leveling with dynamic $\beta^{*}$ squeeze, the sensitivity of the average luminosity to the minimum $\beta^{*}$ permitted by the IR optics greatly depends on the chosen number of events per crossing, as is illustrated in Fig. 4 for LPA.
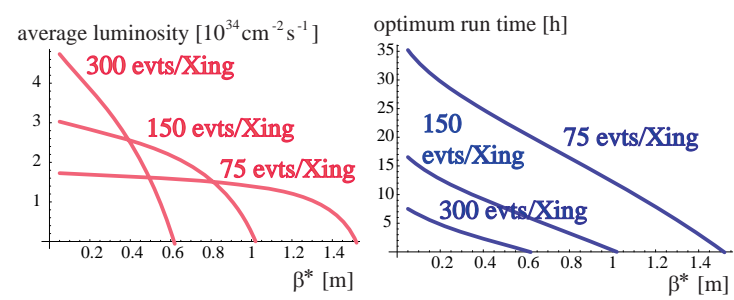

Figure 4. Average luminosity (left) and run time (right) vs. final $\beta^{*}$ for LPA with $\beta^{*}$ leveling.

\section{HIGHER-ENERGY COLLIDER}

A strong physics case calls for higher beam energies. The 2001 design study of a Very Large Hadron Collider (VLHC) [15] with a circumference of $233 \mathrm{~km}$ foresees a staged construction. The first stage would use inexpensive 2-T NbTi 'transmission-line' dipole magnets and reach 40- 
TeV center-of-mass energy, while the second stage based on 10- $\mathrm{T} \mathrm{Nb}_{3} \mathrm{Sn}$ magnets would provide a c.m. energy of $175 \mathrm{TeV}$. The staged construction in the same tunnel is a time-tested formula for success, see e.g. the FNAL Main Ring and the Tevatron, or LEP and LHC. At both stages, the VLHC design peak luminosity is similar to that of the nominal LHC. The main cost driver for stage 1 is the underground civil engineering. The key R\&D items for stage 2 are the 10-T magnets, the vacuum system with large radiated power, and photons stops. A dipole field of about $10 \mathrm{~T}$ was found to be best for beam dynamics reasons. Several designs exist for high-field s.c. dipole magnets based on $\mathrm{Nb}_{3} \mathrm{Sn}$. The optimum size of the cooling pipe and of the beam-screen temperature depend on the synchrotron radiation power emitted per unit length. Room-temperature photon stops will decrease the refigerator power and permit reaching higher energy and luminosity [17]. These photon stops are practical only in a ring with large circumference like the VLHC.

An LHC energy doubler would require dipole magnets with a design field around $19 \mathrm{~T}$, including some margin. 10-14 T fields were demonstrated in several 1-m long $\mathrm{Nb}_{3} \mathrm{Sn}$ dipole models [16]. At LBNL a proof-of-principle magnet with a small 10-mm aperture even reached 16 $\mathrm{T}[16,18]$. CARE-NED [19] aims at developing a large-aperture (up to $88 \mathrm{~mm}$ ), 15-T dipolemagnet model. Development and subsequent construction of $19-\mathrm{T} \mathrm{Nb}_{3} \mathrm{Sn}$ magnets may require close to 20 years, before an LHC with twice the energy could become reality.

More ambitious would be an LHC energy tripler, for which a design magnet field of about $28 \mathrm{~T}$ is necessary. A 24 or $25-\mathrm{T}$ block-coil dipole is being explored by Texas A\&M University [20]. The proposed magnet design employs high- $T_{c}$ superconductor (Bi-2212) in inner highfield windings and $\mathrm{Nb}_{3} \mathrm{Sn}$ for outer low-field windings. The $>20$-T magnet technology is still to be fully demonstrated and 20 years or more may be needed only for the development.

\section{CONCLUSIONS}

LHC luminosity leveling should be seriously considered as it allows for a more regular flow of events at a moderate decrease in average luminosity. The large-Piwinski angle (LPA) option for the LHC upgrade entails less risk and less uncertainties, but it has the drawback of the larger bunch population. The early-separation (ES) option is an optimal back up until we have gained experience with the real LHC. The 2001 design proposal for a staged VLHC contains many stringent arguments. The operation of an energydoubled LHC at $28 \mathrm{TeV}$ c.m. energy may start at the earliest around 2025. A VLHC stage 1 could turn on at about the same time.

\section{ACKNOWLEDGEMENTS}

Many colleagues contributed to the ideas presented in this paper, which we dedicate to the memory of F. Ruggiero (1957-2007).

\section{REFERENCES}

1. J. Strait, private communication (2003).

2. O. Brüning et al, LHC-PROJECT-Report626 (2002).

3. http://esgard.lal.in2p3.fr/Project/Activities/Current/

4. http://care-hhh.web.cern.ch/CARE-HHH/

5. http://www.agsrhichome.bnl.gov/LARP/

6. F. Ruggiero et al, PRST-AB 5, 061001 (2002).

7. E. Todesco et al, PAC'07, Albuquerque.

8. E. Laface et al, private communication (2007)

9. R. Calaga et al, PAC'07, Albuquerque.

10. U. Dorda et al, PAC'07, Albuquerque.

11. O. Brüning et al., LHC-PROJECT-Report1008 (2007).

12. P. Raimondi, PAC'07, Albuquerque.

13. S. Fartoukh, LHC MAC, 16.06.2006.

14. G. Sterbini et al, LHC-Project-Note-403 (2007).

15. G. Ambrosio et al, FNAL-TM-2149 (2001).

16. S. Caspi et al, PAC'05, Knoxville, 107 (2005).

17. P. Bauer et al, PAC'01, Chicago, 3156 (2001).

18. LBNL S.c. Magnet Program Newsletter, no. 2 (2003).

19. http://lt.tnw.utwente.nl/project.php? projectid $=9$

20. P. McIntyre et al, PAC'05, Knoxville (2005). 